\title{
Gene selection and cancer type classification of diffuse large-B-cell lymphoma using a bivariate mixture model for two-species data
}

\author{
Yuhua Su ${ }^{*}$, Dahlia Nielsen ${ }^{2}$, Lei Zhu ${ }^{3}$, Kristy Richards ${ }^{4}$ Steven Suter ${ }^{5}$, Matthew Breen ${ }^{6}$, \\ Alison Motsinger-Reif ${ }^{7}$ and Jason Osborne ${ }^{7}$
}

\begin{abstract}
A bivariate mixture model utilizing information across two species was proposed to solve the fundamental problem of identifying differentially expressed genes in microarray experiments. The model utility was illustrated using a dog and human lymphoma data set prepared by a group of scientists in the College of Veterinary Medicine at North Carolina State University. A small number of genes were identified as being differentially expressed in both species and the human genes in this cluster serve as a good predictor for classifying diffuse large-B-cell lymphoma (DLBCL) patients into two subgroups, the germinal center B-cell-like diffuse large B-cell lymphoma and the activated B-cell-like diffuse large B-cell lymphoma. The number of human genes that were observed to be significantly differentially expressed (21) from the two-species analysis was very small compared to the number of human genes (190) identified with only one-species analysis (human data). The genes may be clinically relevant/important, as this small set achieved low misclassification rates of DLBCL subtypes. Additionally, the two subgroups defined by this cluster of human genes had significantly different survival functions, indicating that the stratification based on gene-expression profiling using the proposed mixture model provided improved insight into the clinical differences between the two cancer subtypes.
\end{abstract}

Keywords: Mixture models, Gene expression, Homology, Lymphoma

\section{Introduction}

Diffuse large-B-cell lymphoma (DLBCL), the most common type of non-Hodgkin lymphoma in adults, accounts for $30 \%$ to $40 \%$ of newly diagnosed lymphomas and has an annual incidence in America of more than 25,000 cases. Combination chemotherapy has transformed DLBCL from a fatal disease into one that is often curable, but only approximately $50 \%$ of all patients are cured [1,2]. This suggests that DLBCL actually comprises several subgroups that differ in responsiveness to chemotherapy. The attempts to define subgroups of DLBCL have often failed due to diagnostic discrepancies. Clinically, the International Prognostic Index (IPI) [3] has been developed for use in the design of future therapeutic trials in patients with aggressive non-Hodgkin lymphoma and in

\footnotetext{
*Correspondence: yhsejo@gmail.com

1 Dr. Su's Statistics, Department of Human Nutrition, Food, and Animal

Sciences, University of Hawaii at Manoa, Honolulu, HI 96822, USA

Full list of author information is available at the end of the article
}

the selection of appropriate therapeutic approaches for individual patients. However, IPI has not been used successfully to predict outcomes in DLBCLs so that patients can be stratified correctly for therapeutic trials. This may be attributed to the fact that the clinical factors of IPI (age, tumor stage, serum lactate dehydrogenase concentration, performance status, and number of extranodal disease sites) neither provide molecular insight into the heterogeneity of DLBCL nor identify specific therapeutic targets $[4,5]$.

Recent developments in microarray technology allow researchers to accurately and precisely measure gene expression patterns in lymphomas which provides the opportunity to revolutionize the way these tumors are grouped and treated. In other words, studying gene expression profiles in lymphomas may provide the opportunity to identify pathways on which the tumor depends and to target the pathways for the development of new drugs. Indeed, gene-expression profiling studies have distinguished three molecular subtypes of DLBCL: 
germinal-center B-cell-like (GCB) DLBCL, activated Bcell-like $(\mathrm{ABC}) \mathrm{DLBCL}$, and primary mediastinal B-cell lymphoma (PMBL) [2,5-8].

The first attempt at examining gene expression profiling to identify distinct B-cell malignancies was made by Alizadeh et al. [9]. A hierarchical clustering algorithm [10] was used to group genes on the basis of similarity of their expression over all subjects. Subjects were also grouped based on the similarities in gene expression using the same clustering method. Two distinct subgroups of DLBCL were found based on the gene expression analysis: GCB DLBCL and ABC DLBCL. Alizadeh et al. [9] discovered that almost all genes that defined GCB DLBCL were highly expressed in normal germinal center $B$ cells and, by contrast, most genes that defined ABC DLBCL were not expressed in normal germinal center $B$ cells. In addition, there was a substantial and significant difference in the average five-year survival rate between patients with GCB DLBCL and ABC DLBCL.

Inspired by the work of Alizadeh [9], Rosenwald et al. [5] found that most of the genes with expression patterns that correlated with survival of the DLBCL subgroups fell within four gene-expression signatures. A gene-expression signature is a group of genes expressed in a specific cell lineage or stage of differentiation or during a particular biologic response and hence genes within the same geneexpression signature are probably associated with similar biologic aspects of tumor [5]. The authors in [5] then developed a molecular predictor consisting of 17 genes for the likelihood of survival after chemotherapy according to gene expression profiles of lymphomas. Shipp et al. [4] adopted the weighted-voting algorithm [11] to develop an outcome predictor with 13 genes and were able to classify two categories of DLBCL patients with very different fiveyear overall survival rates. Note that there is no overlap among the genes in the models derived in [4] and [5].

Wright et al. [8] formulated a DLBCL subgroup predictor based on Bayes' rule, applied this method to the DLBCL gene expression data in [5], and constructed a 27-gene DLBCL subgroup predictor. Next, a new predictor including 14 genes among the previous predictor was constructed and applied to another set of gene expression data from DLBCLs [4]. Wright et al. [8] also demonstrated that the proposed algorithm can define cancer subgroups based on gene expression differences regardless of the DNA microarray platforms and could be used clinically to provide diagnostic information as the resulting survival rates were significantly different for the identified GCB and $A B C D L B C L$ subgroups.

A panel of 36 genes whose expression predicts survival in DLBCL was identified by Lossos et al. [1] through literature review. They [1] selected 6 out of the 36 genes by ranking them on the basis of their predictive power for DLBCL survival obtained by univariate analysis. A 6-gene multivariate Cox proportional-hazards regression model for prediction of survival in DLBCL was constructed and applied to the data from [4] and [5]. Lossos et al. [1] concluded that the measurement of the expression of the six genes was sufficient to predict overall survival in DLBCL after stratifying patients into different risk groups based on their IPI score.

More recently, Blenk et al. [12] analyzed an enlarged data set (original data were generated by [5]) to confirm that there are clear expression differences between $A B C$ and GCB DLBCL. To detect differentially expressed genes, they [12] used limma in [13] and further determined 50 best separating genes for class discovery. An optimal classifier with only 18 genes for distinguishing DLBCL subgroups was conducted. In addition, an optimal molecular survival predictor with only six genes was obtained. However, there was no overlap among the genes used in the classifier and the survival predictor established in [12].

Models introduced in $[1,4,5,8,9,12]$ can be used to distinguish the subgroups in DLBCL and identify rational targets for research into treatment intervention. Moreover, the predictor identified by each study involved only a small number of genes and thus the needed DNA microarrays may be easily developed for clinical prediction. Nonetheless, genes seldom overlap in these models. Blenk et al. [12] showed that 6 of the 18 genes used in the optimal classifier were found again after analyzing another data set from [4]. However, none of these genes were identified in a subsequent investigation of survival [12].

Due to technical differences, the composition of the microarrays used, and the different algorithms used for constructing predictive models, it remains unclear which method and which model best captures the molecular and clinical heterogeneity of diffuse large-B-cell lymphoma. Therefore, the goal in this research was to give an example of how bivariate data can be used for clinical research.

\section{Methods}

Let $X_{a_{i j}}$ and $X_{h_{i l}}$ denote gene expression measurements from the $i^{\text {th }}$ orthologous gene pair [14] for the $j^{\text {th }}$ animal and the $l^{\text {th }}$ human. The following independent linear models describe the association between gene expression and treatment (cancer type):

$$
\begin{aligned}
& X_{a_{i j}}=\beta_{0 a_{i}}+\beta_{1 a_{i}} T_{a_{j}}+e_{a_{i j}}, \\
& X_{h_{i l}}=\beta_{0 h_{i}}+\beta_{1 h_{i}} T_{h_{l}}+e_{h_{i l}},
\end{aligned}
$$

where $T_{a_{j}}$ and $T_{h_{l}}$ are $\{0,1\}$ treatment indicators, and $e_{a_{i j}}$ and $e_{h_{i l}}$ are independent $N\left(0, \sigma_{a}^{2}\right)$ and $N\left(0, \sigma_{h}^{2}\right)$ random variables. The $\sigma_{a}^{2}$ and $\sigma_{h}^{2}$ are variances for $e_{a i j}$ and $e_{h_{i l}}$, respectively. A given gene can be classified as nondifferentially expressed (NDE, showing no signs of treatment effects), positively differentially expressed (pDE, 
showing positive treatment effects), or negatively differentially expressed (nDE, showing negative treatment effects). Furthermore, we assume dependency between differentially expressed orthologs. Therefore, for a human and animal gene pair, there are nine possibilities for categorizing this pair of genes, illustrated in Table 1.

The following two-species nine-component bivariate normal mixture model in $[15,16]$ is proposed to simultaneously model the vectors of the estimated treatment effects $\left(\hat{\beta}_{1 a_{i}}, \hat{\beta}_{1 h_{i}}\right)^{T}$ obtained from Equations 1 and 2:

$$
\begin{gathered}
\left(\begin{array}{c}
\hat{\beta}_{1 a_{i}} \\
\hat{\beta}_{1 h_{i}}
\end{array}\right) \sim \pi_{0} N\left(\left(\begin{array}{c}
0 \\
0
\end{array}\right),\left(\begin{array}{cc}
\sigma_{a 0}^{2} & 0 \\
0 & \sigma_{h 0}^{2}
\end{array}\right)\right) \\
+\pi_{1} N\left(\left(\begin{array}{c}
\mu_{a 1} \\
\mu_{h 1}
\end{array}\right),\left(\begin{array}{cc}
\sigma_{a 1}^{2} & \rho_{1} \sigma_{a 1} \sigma_{h 1} \\
\rho_{1} \sigma_{a 1} \sigma_{h 1} & \sigma_{h 1}^{2}
\end{array}\right)\right) \\
+\pi_{2} N\left(\left(\begin{array}{c}
\mu_{a 2} \\
\mu_{h 2}
\end{array}\right),\left(\begin{array}{cc}
\sigma_{a 2}^{2} & \rho_{2} \sigma_{a 2} \sigma_{h 2} \\
\rho_{2} \sigma_{a 2} \sigma_{h 2} & \sigma_{h 2}^{2}
\end{array}\right)\right) \\
+\pi_{3} N\left(\left(\begin{array}{c}
\mu_{a 3} \\
\mu_{h 3}
\end{array}\right),\left(\begin{array}{cc}
\sigma_{a 3}^{2} & \rho_{3} \sigma_{a 3} \sigma_{h 3} \\
\rho_{3} \sigma_{a 3} \sigma_{h 3} & \sigma_{h 3}^{2}
\end{array}\right)\right) \\
+\pi_{4} N\left(\left(\begin{array}{c}
\mu_{a 4} \\
\mu_{h 4}
\end{array}\right),\left(\begin{array}{cc}
\sigma_{a 4}^{2} & \rho_{4} \sigma_{a 4} \sigma_{h 4} \\
\rho_{4} \sigma_{a 4} \sigma_{h 4} & \sigma_{h 4}^{2}
\end{array}\right)\right) \\
+\pi_{5} N\left(\left(\begin{array}{c}
0 \\
\mu_{h 5}
\end{array}\right),\left(\begin{array}{cc}
\sigma_{a 5}^{2} & 0 \\
0 & \sigma_{h 5}^{2}
\end{array}\right)\right) \\
+\pi_{6} N\left(\left(\begin{array}{c}
0 \\
\mu_{h 6}
\end{array}\right),\left(\begin{array}{cc}
\sigma_{a 6}^{2} & 0 \\
0 & \sigma_{h 6}^{2}
\end{array}\right)\right) \\
+\pi_{7} N\left(\left(\begin{array}{c}
\mu_{a 7} \\
0
\end{array}\right),\left(\begin{array}{cc}
\sigma_{a 7}^{2} & 0 \\
0 & \sigma_{h 7}^{2}
\end{array}\right)\right) \\
+\pi_{8} N\left(\left(\begin{array}{c}
\mu_{a 8} \\
0
\end{array}\right),\left(\begin{array}{cc}
\sigma_{a 8}^{2} & 0 \\
0 & \sigma_{h 8}^{2}
\end{array}\right)\right),
\end{gathered}
$$

\begin{tabular}{|c|c|c|c|}
\hline Category & $\left(\beta_{1 a_{i}}, \beta_{1 h_{i}}\right)$ & $\left(\mu_{\beta_{1 a_{i}}}, \mu_{\beta_{1 h_{i}}}\right)$ & $\operatorname{Corr}\left(\beta_{\boldsymbol{1 a}_{\boldsymbol{i}^{\prime}}} \boldsymbol{\beta}_{\boldsymbol{1} \boldsymbol{h}_{i}}\right)$ \\
\hline 0 & (NDE,NDE) & $(0,0)$ & 0 \\
\hline 1 & (pDE,pDE) & $(+,+)$ & $\rho_{1}$ \\
\hline 2 & $(\mathrm{nDE}, \mathrm{nDE})$ & $(-,-)$ & $\rho_{2}$ \\
\hline 3 & (pDE,nDE) & $(+,-)$ & $\rho_{3}$ \\
\hline 4 & (nDE,pDE) & $(-,+)$ & $\rho_{4}$ \\
\hline 5 & (NDE,pDE) & $(0,+)$ & 0 \\
\hline 6 & (NDE,nDE) & $(0,-)$ & 0 \\
\hline 7 & (pDE,NDE) & $(+, 0)$ & 0 \\
\hline 8 & (nDE,NDE) & $(-, 0)$ & 0 \\
\hline
\end{tabular}

where $\pi_{k}, k=0, \ldots, 8$ denote the mixing weights (the probability that an observation belongs to the $k$ th component). Note that $\sum_{k=0}^{8} \pi_{k}=1$ and $\pi_{k} \geq 0 .\left(\mu_{a k}, \mu_{h k}\right)^{T}$ and $\left(\sigma_{a k}^{2}, \sigma_{h k}^{2}\right)^{T}$ are the vectors of the means and variances, respectively, for each species in each mixture component. $\rho_{k}$ denotes the correlation between orthologs under the $k$ th category. To accommodate the possible patterns of

Table 1 Possible categories of $\left(\beta_{1 a_{i}}, \beta_{1 h_{i}}\right)^{T}$ the gene expression for animal and human due to treatment intervention (different cancer types), the following constraints are imposed: $\mu_{a 1} \geq 0, \mu_{h 1} \geq 0, \mu_{a 2} \leq 0$, $\mu_{h 2} \leq 0, \mu_{a 3} \geq 0, \mu_{h 3} \leq 0, \mu_{a 4} \leq 0, \mu_{h 4} \geq 0, \mu_{h 5} \geq 0$, $\mu_{h 6} \leq 0, \mu_{a 7} \geq 0$, and $\mu_{a 8} \leq 0 ; \rho_{0}=0, \rho_{5}=0, \rho_{6}=0$, $\rho_{7}=0$, and $\rho_{8}=0$.

Gene membership is determined according to the maximum posterior probability that an observation $\left(\hat{\beta}_{1 a_{i}}, \hat{\beta}_{1 h_{i}}\right)^{T}$ comes from the $k^{\text {th }}$ component of the mixture.

A parametric bootstrap method in $[17,18]$ to estimate the standard errors for the estimated parameters is provided. Bootstrapping is the practice of estimating properties of an estimator by measuring those properties when sampling from an approximating distribution. The basis of the bootstrap methodology is simple. In the parametric bootstrap setting, consider $F$ to be a member of some prescribed parametric family and obtain $\widehat{F}_{n}$ by estimating the family parameters, in this case, $\left(\pi_{k}, \mu_{a k}, \mu_{h k}, \Sigma_{k}\right)^{T}$, $k=0, \ldots, 8$, from the data. In each iteration, by generating an iid random sequence, called a 'resample' from the distribution $\widehat{F}_{n}$, new estimates of the parameters are obtained and the sampling properties (such as the mean, standard deviation, bias, and shape) can be evaluated.

The procedure of the parametric bootstrap resampling method to obtain the estimated standard errors of the estimated parameters for the nine-component mixture model is described as follows:

1. $\widehat{F}_{n}$ is formed by substituting the estimates of $\left(\mu_{a k}, \mu_{h k}\right)^{T}$ and $\Sigma_{k}$ into the 9-component mixture model (3).

2. The numbers of genes in category 0 through category $8\left(n_{0}, n_{1}, \ldots, n_{8}\right)$ are drawn from a multinomial distribution with parameters $n$ and $\mathbf{p} \cdot n$ is the number of trials for each multinomial random variable. In this study, it is equal to the number of orthologs in two-species data. $\mathbf{p}$ is the vector of event probabilities for each trial. In this study, $\mathbf{p}$ is the vector of the mixing weights estimated from the data. The new mixing weights are then calculated for the bootstrap resampling and plugged into the nine-component mixture model (Equation 3 ) to form $\widehat{F}_{n}$.

3. Bootstrap samples $\left(\hat{\beta}_{1 a_{i}}^{*}, \hat{\beta}_{1 h_{i}}^{*}\right)^{T}$ of size $n$ are drawn from $\widehat{F}_{n}$ formed above.

4. For each bootstrap resampling, obtain the numerically approximated maximum likelihood estimates for the parameters in the nine-component mixture using the expectation-maximization (EM) algorithm.

5. Repeat steps 1 to $4 B$ times independently. $B$ is the number of bootstrap replications. Calculate the 
empirical standard deviation of a series of bootstrap replications of $\hat{\theta}$ accordingly. $\hat{\theta}$ is the estimator of $\theta$, the parameter of interest. Since the standard error of the mean $(s / \sqrt{n}$, sample standard deviation divided by the squared root of the size of the sample) is the estimate of the true standard deviation of the sample mean $(\sigma / \sqrt{n}$, standard deviation for the population divided by the squared root of the size of the sample), essentially, the standard deviation of the bootstrap estimator obtained here is an estimation of the standard error of the mean for the parameter of interest. The bootstrap standard error $\widehat{S E}_{B}$ of $\hat{\theta}$ is calculated as follows:

$$
\widehat{S E}(\hat{\theta})_{B}=\sqrt{\frac{1}{B-1} \sum_{b=1}^{B}\left(\hat{\theta}_{b}^{*}-\overline{\hat{\theta}}^{*}\right)^{2}},
$$

where $\hat{\theta}_{b}^{*}$ is the estimator of $\theta$ calculated from the $b^{\text {th }}$ bootstrap resample $(b=1, \ldots, B), \overline{\hat{\theta}}^{*}=\sum_{b=1}^{B} \hat{\theta}_{b}^{*} / B$; $B$ is the total number of resamples (each of size $n$ ) collected from the empirical estimate of $F$.

\section{Data sources}

In order to improve treatments for non-Hodgkin lymphoma in human and canine patients, researchers from North Carolina State University's College of Veterinary Medicine and the University of North Carolina at Chapel Hill Lineberger Comprehensive Cancer Center conducted research to study tissue samples from human and canine non-Hodgkin lymphoma patients, with the hope of creating a genomic profile of non-Hodgkin lymphoma that would give oncologists and veterinarians greater insight into the disease's biology and obtain the information that could lead to a clinical benefit for both species. The study protocol was approved by the Institutional Animal Care and Use Committee of North Carolina State University.

The team recruited dogs diagnosed with lymphoma to collect tissue samples for study. The dog data were measured at the probe set level on Affymetrix Canine Genome 2.0 array (Canine_2, Affymetrix Inc., Santa Clara, CA, USA), with a total number of probe sets equal to 43,035 . Forty-eight dogs with one of the following diagnostic results were recruited: B-cell lymphoma (27 dogs), T-cell lymphoma (10 dogs), B-cell acute lymphoblastic leukemia (1 dog), T-cell acute lymphoblastic leukemia (4 dogs), and normal (6 dogs). Among the 27 dogs with B-cell lymphoma, 14 of them were diagnosed histopathologically with DLBCL. The 14 DLBCL patients could be further divided into two subgroups: 5 ABC DLBCL patients and 9 GCB DLBCL patients. For the purpose of this research, only data for the 14 dogs with DLBCLs were used. The dog microarray gene expression data were LOESS normalized by JMP Genomics 4.0 (Cary, NC, USA).

Corresponding data for human patients with lymphoma were extracted from the Gene Expression Omnibus (GEO) database [19]. Data for 460 lymphoma patients were retrieved from two series with GEO accession number: GSE10846 [7] and GSE11318 [6]. The human data were measured at the probe set level on Affymetrix Human Genome U133 2.0 array (HG-U133_Plus_2), with a total number of probe sets equal to 54,675. The human microarray gene expression data were also LOESS normalized by JMP Genomics 4.0. Based on the gene expression, two distinct subgroups were identified after principle component analysis. This implied that there may be a strong batch effect among the samples. Hence, only samples from one of these two subgroups were included in the data analysis. This resulted in 219 human subjects consisting of 31 PMBL, 78 ABC DLBCLs, 80 GCB DLBCLs, and 29 unclassified DLBCLs (distinguishing between subgroups of DLBCL is through gene-expression profiling $[6,7])$. To make the animal and human data comparable, only data for $\mathrm{ABC}$ and GCB DLBCLs with corresponding survival information were used. This resulted in a final dataset with $77 \mathrm{ABC}$ DLBCL patients and 79 GCB DLBCL patients.

After averaging probe sets across a gene to obtain a gene-level transcript value, the orthologous information from HomoloGene release 64 at website ftp://ftp.ncbi.nih. gov/pub/HomoloGene/build64/ was applied to acquire the mappings between dog and human. This led to a total of 6,566 pairs of dog and human orthologs.

\section{Data analysis}

We recall that the objective of the data analysis was to identify rational targets (genes) for treatment intervention simultaneously for both dog and human lymphoma patients with the hope of giving researchers greater insight into the disease's biology. Furthermore, it was also of interest to verify if the targeted genes can serve as a good predictor to clinically distinguish subgroups of DLBCL in humans. Because the samples in humans that were used to estimate the distribution of the bivariate mixture model were also used to build the classification function, there was a possibility of over-fitting, resulting in a model that would indicate an over-optimistic separation between the subgroups than would be found in independent data. To avoid the biased classification result, a leave-one-out-cross-validation (LOOCV) procedure [20] was introduced as the following steps:

1. Use all 14 observations for dogs and obtain the estimated coefficients of cancer type effect on gene 
expression $\hat{\beta}_{1 a_{i}}, i=1, \ldots, 6,566$. Omit one observation from the 156 human observations and obtain the estimated coefficients of cancer type effect $\hat{\beta}_{1 h_{i}}$.

2. Use all $\left(\hat{\beta}_{1 a_{i}}, \hat{\beta}_{1 h_{i}}\right)^{T}$ to construct the nine-component bivariate normal mixture model. Identify gene membership accordingly.

3. Use genes classified into categories (1, 2, 3, and 4) (differentially expressed in both species) to develop a classification rule based on the remaining 155 human observations. Develop another classification rule based on genes classified into categories $(1,2,3,4,5$, and 6) (differentially expressed in human).

4. For the purpose of comparison, identify differentially expressed human genes by performing a single species analysis for human only. Choose genes based on the $p$ values of the $t$ statistics after adjusting for multiple comparison by controlling the false discovery rate (FDR) [21] at levels 0.01 and 0.00001 .

5. Classify the holdout human observation using the classification rules constructed in steps 3 and 4 .

6. Repeat steps 1, 2, 3, 4 and 5 until every one of the human observations is classified.

The classification rule was established through the $M$ dimensional centroid obtained from the $k$-means [22] clustering process applied to the training set. $M$ was the number of genes retained for performing cancer type classification. $k$ was equal to 2 as there were two types of cancer. SAS PROC FASTCLUS [23] was used to carry out the $k$-means clustering.

Since $k$-means does the clustering, and not the classification, the class of each cluster has to be determined for the classification rule before it can be used to classify future observations. Table 2 demonstrates the results of the $k$-means clustering.

Clusters were designated so that the minimum misclassification rate was achieved. Therefore,

$$
\begin{cases}\text { if } & n_{12}+n_{21}<n_{22}+n_{11} \\ \Rightarrow & \text { cluster } 1=\text { "ABC DLBCL" and cluster } 2=\text { "GCB DLBCL", } \\ \text { if } & n_{12}+n_{21}>n_{22}+n_{11} \\ \Rightarrow & \text { cluster } 1=\text { "GCB DLBCL" and cluster } 2=\text { "ABC DLBCL", } \\ \text { if } \quad n_{12}+n_{21}=n_{11}+n_{22} \\ \Rightarrow \text { randomly assign cluster labels. }\end{cases}
$$

Table 2 The $k$-means clustering results

\begin{tabular}{lcc}
\hline & Cluster 1 & Cluster 2 \\
\hline ABC DLBCL & $n_{11}$ & $n_{12}$ \\
GCB DLBCL & $n_{21}$ & $n_{22}$ \\
\hline
\end{tabular}

The classification rule established at the $l^{\text {th }} \mathrm{LOOCV}$ procedure was then

$\left\{\begin{array}{l}\text { if } \sum_{m=1}^{M_{l}}\left(x_{h_{m l}}-\mu_{l, 1 m}^{\text {centroid }}\right)^{2}<\sum_{m=1}^{M_{l}}\left(x_{h_{m l}}-\mu_{l, 2 m}^{\text {centroid }}\right)^{2} \\ \Rightarrow \text { classify the } l \text { th human subject into cluster } 1, \\ \text { else } \Rightarrow \text { classify the } l^{\text {th }} \text { human subject into cluster } 2 .\end{array}\right.$

$M_{l}$ was the total number of genes retained at the $l^{\text {th }}$ LOOCV procedure for cancer type classification. $l=$ $1, \ldots, 156$, as there were 156 human DLBCL patients. $x_{h_{m l}}$ was the $m^{\text {th }}$ gene expression for the $l^{\text {th }}$ hold-out human subject. For the $l^{\text {th }}$ LOOCV procedure, $\mu_{l, 1 m}^{\text {centroid }}$ and $\mu_{l, 2 m}^{\text {centroid }}$ were the $m^{\text {th }}$ centroid means $\left(m=1, \ldots, M_{l}\right)$ calculated from the $k$-means $(k=2)$ algorithm for cluster 1 and cluster 2 , respectively.

\section{Results}

\section{Parameter estimation}

The maximum likelihood estimates of the parameters in the nine-component bivariate normal mixture model computed using the EM algorithm [24] are given in Table 3. The estimated mixture weight for category 0 was $\hat{\pi}_{0}=0.823$ indicating approximately $5,404(6,566 \times$ 0.823) pairs of uninteresting dog and human orthologs. $\left(\mu_{a}, \mu_{h}\right)^{T}$ denotes the mean vector of each mixture component. Most of the estimated mean vectors were slightly larger in categories 1 through 4 than those in categories 5 through 8. It appeared that the magnitude of the estimated difference of expression in genes related to lymphoma in both species tended to be larger than in genes where differential expression was exhibited in only one species.

\section{Gene selection and cancer type classification}

For the 156 LOOCV instances, the proposed mixture model determined 21 (14 genes appearing in the intersection of all hold-outs) human genes in categories $(1,2,3$, and 4) and 279 (185 genes appearing in the intersection of all hold-outs) human genes in categories $(1,2,3,4,5$, and 6). While analyzing the human data alone and controlling the FDR at levels 0.01 and 0.00001, 935 (706 genes appearing in the intersection of all hold-outs) and 190 (139 genes appearing in the intersection of all hold-outs) genes were identified as differentially expressed, respectively. Figure 1 shows the scatter plots of $\left(\hat{\beta}_{1 a}, \hat{\beta}_{1 h}\right)^{T}$ for all orthologs and the 21 pairs of orthologs in categories $(1,2,3$, and 4$)$; i.e., the genes in this group showed evidence of distinguishing the two types of cancer for both species. Clearly, most of the pairs scattered around the $(0,0)$ origin, indicating that these genes (for both species) did not have potential for serving as markers that could distinguish the two subgroups of DLBCL.

After identifying human genes that showed signs of discriminating between ABC DLBCL and GCB DLBCL and 
Table 3 Summary of parameter estimates for the bivariate mixture model averaged over the 156 LOOCV outcomes

\begin{tabular}{|c|c|c|c|c|c|c|}
\hline \multirow[b]{2}{*}{ Category } & \multicolumn{6}{|c|}{ Parameter } \\
\hline & $\pi_{k}$ & $\mu_{a k}$ & $\mu_{h k}$ & $\sigma_{a k}^{2}$ & $\rho_{k} \sigma_{a k} \sigma_{h k}$ & $\sigma_{h k}^{2}$ \\
\hline 0 & $0.823(0.077)$ & $\mathrm{NE}$ & NE & $0.013(0.001)$ & $\mathrm{NE}$ & $0.012(0.001)$ \\
\hline 1 & $0.001(0.005)$ & $0.341(0.081)$ & $0.022(0.130)$ & $0.004(0.010)$ & $0.001(0.017)$ & $0.022(0.060)$ \\
\hline 2 & $0.001(0.001)$ & $-0.602(0.243)$ & $-0.831(0.193)$ & $0.199(0.082)$ & $-0.128(0.048)$ & $0.089(0.036)$ \\
\hline 3 & $0.001(0.004)$ & $0.495(0.195)$ & $-0.564(0.244)$ & $0.042(0.078)$ & $-0.042(0.041)$ & $0.119(0.074)$ \\
\hline 4 & $0.000(0.003)$ & $-1.131(0.422)$ & $0.758(0.305)$ & $0.000(0.028)$ & $0.000(0.015)$ & $0.000(0.050)$ \\
\hline 5 & $0.020(0.008)$ & $\mathrm{NE}$ & $0.492(0.121)$ & $0.020(0.021)$ & $\mathrm{NE}$ & $0.058(0.049)$ \\
\hline 6 & $0.011(0.004)$ & $\mathrm{NE}$ & $-0.517(0.136)$ & $0.034(0.093)$ & $\mathrm{NE}$ & $0.040(0.044)$ \\
\hline 7 & $0.130(0.077)$ & $0.331(0.038)$ & $N E$ & 0.018(0.009) & $\mathrm{NE}$ & $0.025(0.001)$ \\
\hline 8 & $0.012(0.008)$ & $-0.478(0.339)$ & NE & $0.011(0.051)$ & $\mathrm{NE}$ & $0.042(0.054)$ \\
\hline
\end{tabular}

Numbers in parentheses are the bootstrap standard errors; $B=5,000$ (the number of bootstrap replications); NE not estimated.

based either on two-species analysis (the nine-component bivariate mixture model) or on single-species (human only) analysis, the next step in the LOOCV procedure was to classify the hold-out human subject according to a classification rule established using the same set of genes. Table 4 summarizes the classification results over the 156 LOOCV instances under the four different gene selection criteria. It was interesting to see that using human genes from categories (1, 2, 3, and 4), categories $(1,2,3,4$, 5 , and 6) (two-species analysis), and the genes selected by controlling FDR at 0.00001 (single-species analysis) gave a very similar number of misclassifications, 17, 16, and 19, respectively. Nonetheless, choosing FDR $=0.01$, the gene list was largely expanded. The enlarged gene

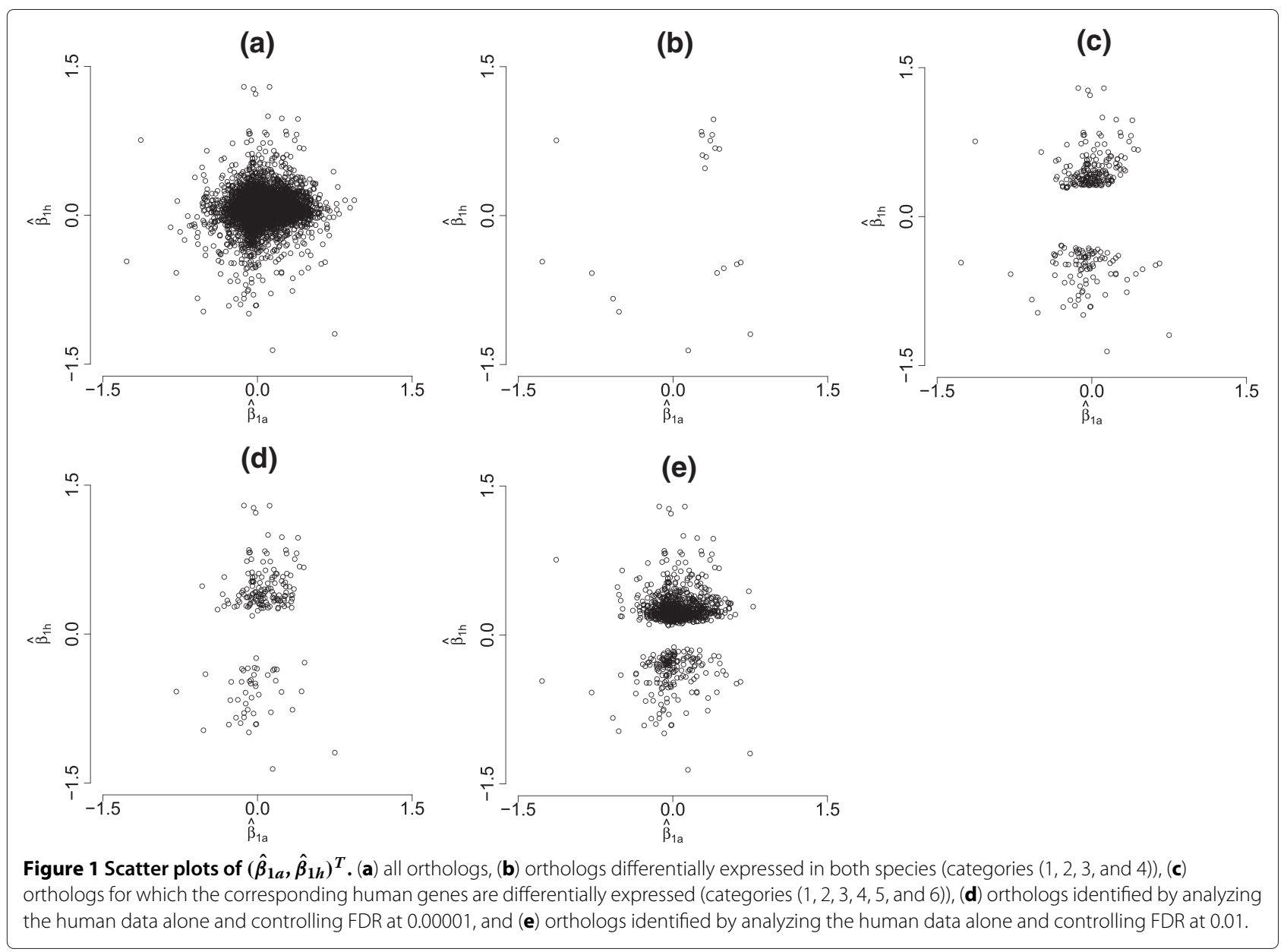


Table 4 Misclassification tables using different criteria

\begin{tabular}{|c|c|c|c|c|c|c|c|c|}
\hline \multirow[b]{2}{*}{ Model prediction/subgroup } & \multicolumn{2}{|c|}{ Categories $(1,2,3$, and 4$)$} & \multicolumn{2}{|c|}{ Categories $(1,2,3,4,5$, and 6$)$} & \multicolumn{2}{|c|}{$\mathrm{FDR}=0.00001$} & \multicolumn{2}{|c|}{$\mathrm{FDR}=0.01$} \\
\hline & $A B C$ & GCB & $A B C$ & GCB & $A B C$ & GCB & $A B C$ & GCB \\
\hline$A B C D L B C L$ & 72 & 5 & 64 & 13 & 58 & 19 & 0 & 77 \\
\hline GCB DLBCL & 12 & 67 & 3 & 76 & 0 & 79 & 2 & 77 \\
\hline
\end{tabular}

list resulted in a very poor classification result: 79 subjects were misclassified, among whom the entire group of ABC DLBCLs were misclassified as GCB DLBCLs. Misclassification rates were $0.109,0.103,0.122$, and 0.506 , accordingly. It was reasonable to conclude that the classification results based on two-species (dog and human) data, in general, may outperform those based on only single-species (human) data.

\section{Prognostic DLBCL sub-categories defined by gene expression profiles}

Does the taxonomy of DLBCL derived from gene expression patterns define clinically distinct subgroups of patients? To confirm that these two DLBCL subgroups defined by gene expression (the 21 genes in categories $(1,2,3$, and 4)) were both biologically and clinically distinct so that the mixture model approach could form the basis of a robust diagnostic test that may prove useful in assessing the results of therapeutic trials in DLBCL, overall survival and subgroup survival based on two types of gene-expression profiling, in [6] and [7] and the proposed mixture model approach, were plotted.

Figure 2 shows the nonparametric Kaplan-Meier survival probability estimates [25] for patients with DLBCL under two situations, unstratified and stratified, by geneexpression profiling. Treating the DLBCL patients regardless of the biological difference between the subgroups gave a 5 -year survival rate of $45 \%$. The 5 -year survival rates after stratifying the patients according to the geneexpression profiling performed in [6] and [7] were 31\% for ABC DLBCL and 59\% for GCB DLBCL as compared with the rates of $29 \%$ for $\mathrm{ABC}$ DLBCL and $64 \%$ for GCB DLBCL if patients were stratified by the gene-expression profiling results based on the proposed nine-component mixture model. Under both types of stratification, $A B C$ and GCB DLBCL were associated with statistically significant differences in overall survival $(p<0.0001)$. A log-rank test [26] was used to test the hypothesis of equal survival functions. The molecular dissection of DLBCL by geneexpression profiling using the proposed nine-component mixture model apparently identified different features of these patients that influence their survival.

As determined by gene-expression profiling performed in [6] and [7], among the 156 patients, there were $77 \mathrm{ABC}$ DLBCLs and 79 GCB DLBCLs. Conversely, the stratification stated by the gene-expression profiling using the proposed nine-component mixture model gave a result of 84 ABC DLBCLs and 72 GCB DLBCLs. More specifically, five of the $A B C$ DLBCLs had been classified as GCB DLBCLs, and 12 of the GCB DLBCLs had been categorized as ABC DLBCLs. However, the difference between the median survival time (years) of the subgroups stratified by gene-expression profiling performed in [6] and [7] was smaller than that of the subgroups stratified by gene expression profiling using the proposed ninecomponent mixture model (8.76 vs. 9.31$)$. This may imply (a)

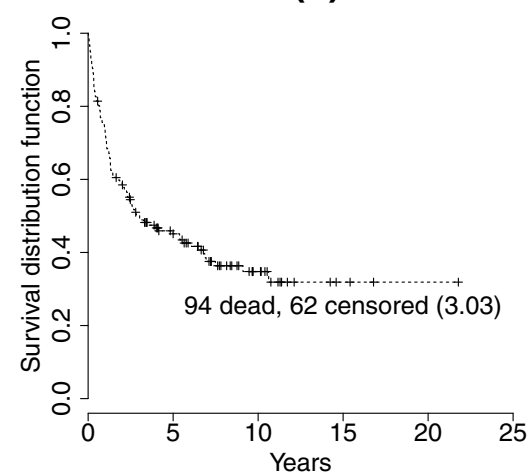

(b)

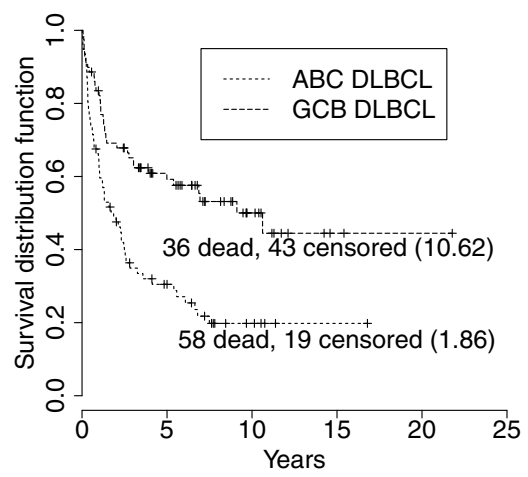

(c)

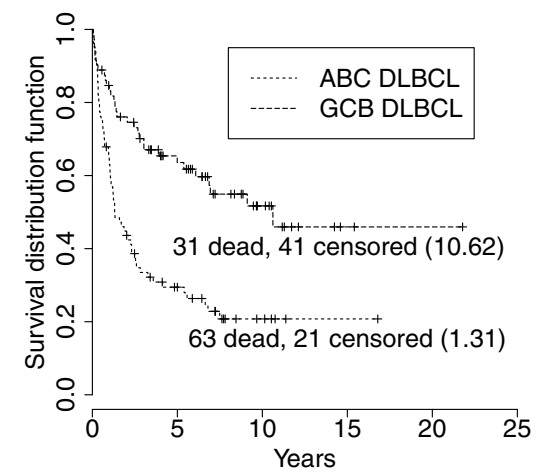

Figure 2 Kaplan-Meier survival probability estimates for the dog and human lymphoma study. (a) No stratification, (b) stratification based on the results of gene-expression profiling performed in [6] and [7], and (c) stratification based on the gene-expression profiling resulted from the proposed nine-component mixture model. Numbers in parentheses are medians. 
that the stratification based on the gene expression profiling using the proposed nine-component mixture model provided better insight for the clinical difference between $A B C$ and GCB DLBCL. These results suggested that the microarray-based outcome predictor not only reflected the clinical difference between the two DLBCL subgroups, but also provided a possible strategy of investigation for further individualization of patient treatment.

\section{Justification of the 21 selected human genes}

To validate the relevance between specific genes and phenotypes, a careful search of the literature was undertaken using Entrez Gene [27]. Some of these 21 genes (the genes in Table 5 with Entrez ID highlighted in italics) were identified by this search as potentially associated with the development of DLBCL. A brief summary of the relationship between these candidate genes and the development of DLBCL is given as follows:

1. CD39 [Entrez Gene:953] is a B lymphocyte activation marker and has powerful functions in the immune system [28].

2. The expression pattern of JAW1 [Entrez Gene:4033], a lymphoid-restricted protein, suggested that this protein may have a role in the developmentally regulated trafficking of the antigen receptors in $\mathrm{B}$ cells and may influence lymphoid development [29]. Tedoldi et al. [30] pointed out that high levels of Jaw1 mRNA were found in germinal center B-cells and in diffuse large B-cell lymphomas of germinal center subtype.

3. The importance of LMO2 [Entrez Gene:4005], though its function in germinal center cells is unknown, as a candidate marker involved in the development of DLBCL has been discussed in several papers. Natkunam et al. [31] studied LMO2 at the protein level and confirmed that LMO2 is expressed specifically in germinal center B cells, which is fully in keeping with gene-expression profiling studies that showed high levels of LMO2 mRNA in germinal center B cells. They in [31] also observed that among DLBCLs, LMO2 tended to be expressed in cases assigned by phenotyping to the GCB categories and can therefore be added to the panel of markers that pathologists may use to subcategorize lymphomas. Morton et al. [32] claimed that LMO2 is one of the candidate genes involved in lymphocyte development and is highly expressed in germinal center lymphocytes. Durnick et al. [33] studied the relationship between $L M O 2$ expression and $t(14 ; 18) / I G H-B C L 2$, a specific marker of lymphomas of germinal center origin and has been specifically associated with the GCB subgroup of DLBCL as determined by gene expression profiling but not in the $\mathrm{ABC}$ cases. There was a statistically significant association between IGH-BCL2 fusion and LMO2 protein expression and hence $L M O 2$ was suggested as a potential marker for the GCB phenotype. A similar conclusion has also been reached by [1].

4. Germinal center B lymphocytes prominently express at least two regulators of G-protein signaling (RGS) proteins, RGS1 and RGS13 [Entrez Gene:6003]. RGS is a family of proteins acting to limit and modulate heterotrimeric G-protein signaling. Han et al. [34] discovered that RGS1 and RGS13 act together to regulate chemokine receptor signaling in human germinal center B lymphocytes. The results provide some insight toward finding methods to reduce or eliminate an organism's negative reaction to a treatment stimulus.

5. The importance of the transcription factor FOXP1 [Entrez Gene:27086] as marker for the activated B-cell-like signature has been well-established $[5,9]$. Banham et al. [35] investigated the prognostic importance of FOXP1 protein expression in DLBCL and found that the overall empirical survival curves for the two subgroups based on the expression of FOXP1 are significantly different. Goatly et al. [36] made an attempt to discover the underlying molecular mechanism of FOXP1 expression in lymphoma development by investigating the FOXP1 translocation, copy number change, and protein expression in mucosa-associated lymphoid tissue lymphoma and DLBCL. Korac and Dominis [37] explored the association between FOXP1, BCL2, and BCL6 gene expression in diffuse large B-cell lymphoma tumor cells. FOXP1 protein was detected in 28 patients; genetic abnormalities involving the FOXP1 locus were found in 19 patients, and both were present in 13 patients, among the samples of lymph nodes from 53 patients with newly diagnosed DLBCL. FOXP1 genetic abnormalities have been found to be associated with both BCL2 and BCL6 expression. Though it has been discovered that BCL2 and BCL6 proteins have an impact on diffuse large Bcell lymphoma development and outcome, they may not be good prognostic markers. FOXP1 has played a role in the development of DLBCL. The identified association among FOXP1, BCL2, and BCL6 indicates the possibilities of uncovering the development process in diffuse large B-cell lymphoma tumor cells. In addition, Nyman et al. [38] used FOXP1 and MUM1/IRF4 as activated B-cell-like markers to distinguish patients between the activated B-cell-like and other diffuse large B-cell lymphoma subtypes. Most recently, six common prognostic biomarkers, including FOXP1, were used to conclusively decide the cut-off values calculated by receiver operating 
Table 5 Summary of the gene-specific information (retrieved from Entrez Gene, an NCBI's database for gene-specific information)

\begin{tabular}{|c|c|c|c|c|c|}
\hline Entrez Gene ID & Category & Dog ortholog & Frequency $^{a}$ & Symbol & Official full name \\
\hline 953 & 1 & 486810 & 156 & $\begin{array}{l}\text { CD39; ATPDase; } \\
\text { FLJ40921; FLJ40959; } \\
\text { NTPDase-1; } \\
\text { DKFZp686D194; } \\
\text { DKFZp686I093; ENTPD1 }\end{array}$ & $\begin{array}{l}\text { Ectonucleoside triphosphate } \\
\text { diphosphohydrolase } 1\end{array}$ \\
\hline 1278 & 2 & 403824 & 156 & $\mathrm{Ol4} ; \mathrm{COL} 1 \mathrm{~A} 2$ & Collagen, type I, alpha 2 \\
\hline 2530 & 1 & 448804 & 156 & MGC26465; FUT8 & $\begin{array}{l}\text { Fucosyltransferase } 8 \text { (alpha }(1,6) \text { fucosyltrans- } \\
\text { ferase) }\end{array}$ \\
\hline 4005 & 2 & 609006 & 156 & $\begin{array}{l}\text { TTG2; RBTN2; RHOM2; } \\
\text { RBTNL1; LMO2 }\end{array}$ & LIM domain only 2 (rhombotin-like 1) \\
\hline 4033 & 3 & 486631 & 156 & JAW1; LRMP & Lymphoid-restricted membrane protein \\
\hline 5919 & 2 & 475532 & 156 & TIG2; HP10433; RARRES2 & $\begin{array}{l}\text { Retinoic acid receptor responder (tazarotene } \\
\text { induced) } 2\end{array}$ \\
\hline 6003 & 3 & 612789 & 124 & MGC17173; RGS13 & Regulator of G-protein signaling 13 \\
\hline 6856 & 3 & 475889 & 154 & SYPL; H-SP1; SYPL1 & Synaptophysin-like 1 \\
\hline 6925 & 1 & 403949 & 156 & $\begin{array}{l}\text { E2-2; ITF2; PTHS; SEF2; } \\
\text { SEF2-1; SEF2-1A; SEF2-1B; } \\
\text { bHLHb19; MGC149723; } \\
\text { MGC149724; TCF4 }\end{array}$ & Transcription factor 4 \\
\hline 7037 & 1 & 403703 & 11 & $\begin{array}{l}\text { TFR; CD71; TFR1; TRFR; } \\
\text { TFRC }\end{array}$ & Transferrin receptor (p90, CD71) \\
\hline 9435 & 1 & 485701 & 156 & $\begin{array}{l}\text { C6ST; GST2; GST-2; Gn6ST- } \\
1 ; \text { CHST2 }\end{array}$ & $\begin{array}{l}\text { Carbohydrate }(\mathrm{N} \text {-acetylglucosamine-6-O) } \\
\text { sulfotransferase } 2\end{array}$ \\
\hline 9760 & 2 & 486964 & 156 & TOX1; KIAA0808; TOX & $\begin{array}{l}\text { Thymocyte selection-associated high mobil- } \\
\text { ity group box }\end{array}$ \\
\hline 10447 & 3 & 612336 & 154 & ILEl; GS3786; FAM3C & Family with sequence similarity 3 , member $C$ \\
\hline 23075 & 3 & 485385 & 4 & $\begin{array}{l}\text { HSPC321; SWAP-70; } \\
\text { FLJ39540; KIAA0640; } \\
\text { SWAP70 }\end{array}$ & $\begin{array}{l}\text { SWAP switching B-cell complex 70kDa } \\
\text { subunit }\end{array}$ \\
\hline 25816 & 1 & 481428 & 156 & $\begin{array}{l}\text { GG2-1; SCCS2; SCC-S2; } \\
\text { MDC-3.13; TNFAIP8 }\end{array}$ & $\begin{array}{l}\text { Tumor necrosis factor, alpha-induced } \\
\text { protein } 8\end{array}$ \\
\hline 27086 & 1 & 484692 & 156 & $\begin{array}{l}\text { QRF1; 12CC4; hFKH1B; } \\
\text { HSPC215; FLJ23741; } \\
\text { MGC12942; MGC88572; } \\
\text { MGC99551; FOXP1 }\end{array}$ & Forkhead box P1 \\
\hline 56941 & 2 & 484628 & 156 & $\begin{array}{l}\text { DC12; MGC111075; } \\
\text { C3orf37 }\end{array}$ & Chromosome 3 open reading frame 37 \\
\hline 81552 & 1 & 608562 & 145 & $\begin{array}{l}\text { ECOP; GASP; FLJ20532; } \\
\text { DKFZp564K0822; VOPP1 }\end{array}$ & $\begin{array}{l}\text { Vesicular, overexpressed in cancer, } \\
\text { prosurvival protein } 1\end{array}$ \\
\hline 81641 & 1 & 479002 & 116 & $\begin{array}{l}\text { Apm; Apn; KZP; AP-M; AP- } \\
\text { N; Lap1; rAPN; Anpep }\end{array}$ & Alanyl (membrane) aminopeptidase \\
\hline 121355 & 4 & 477590 & 156 & $\begin{array}{l}\text { FAM112B; FLJ32942; } \\
\text { GTSF1 }\end{array}$ & Gametocyte specific factor 1 \\
\hline 219972 & 1 & 475960 & 156 & $\begin{array}{l}\text { MPG1; MGC132657; } \\
\text { MGC138435; MPEG1 }\end{array}$ & Macrophage expressed 1 \\
\hline
\end{tabular}


curves to predict survival for DLBCL patients [39]. All these results suggested that FOXP1 expression may be important in DLBCL pathogenesis.

Among the 21 selected genes, five (from categories 1, 2 , and 3) have been carefully examined to explore their association with the development of lymphoma. From the mixture model assumption, genes in the same category should react to the stimulus (drug treatment, cancer type, etc.) in a similar manner. Hence, the implications of these 21 genes (some of them may not have been studied scrupulously) in lymphoma may provide timely and important insight on guiding future investigations of their roles in both B-cell biology and lymphoma development.

\section{Conclusions}

Since the development of high throughput gene expression technology, the important and difficult task of searching for genes that exhibit differences across species (cancer types or treatment groups in drug trials) has been the focus of much research. Simultaneously analyzing gene expression across two species takes into account the biological similarity between different organisms while identifying genes that could be potential prognostic markers and increase the power to detect differences. Identification of the relevant genes and a better understanding of the associated molecular pathways may open new possibilities in cancer diagnosis and treatment. Furthermore, it may become a practical assay for newly diagnosed patients to optimize their clinical management.

In this case study, the application of the proposed nine-component mixture model successfully reduced the quantities of variables (genes) needed to be investigated for the study of two types of DLBCL in humans. The dimension of variables decreased from 6,566 to 21 , a cluster of genes that were identified as being differentially expressed in both species. On the other hand, an analysis of data from one species that selected genes using a specified FDR led to a much longer list of differentially expressed human genes (935 genes with FDR $=0.01$ and 190 with FDR $=0.00001)$. Furthermore, the misclassification rate for human cancer type classification using clustering with gene expression from these 21 genes identified by the bivariate mixture model was remarkably low. The survivorship of the patients stratified according to this clustering was very different across the two types of cancer, indicating that the stratification based on geneexpression profiling using the proposed nine-component mixture model provided better insight for the clinical differences between the two types of cancer.

While validating the relevance of the identified human genes through NCBI's database, literature, if any, for the corresponding dog orthologs were also searched. Far less research about DLBCL has been conducted for canines.
As the model assumption is based on the biological mechanism behind humans and animals, the promising DLBCL classification results based on the human genes may be extended to dogs. Furthermore, currently, direct experiments on humans are not practical. This research provides the possibility for scientists to conduct observational or experimental research on modeling organisms as the first step to understand phenotypes, and then extend the findings to humans for further investigation.

\section{Abbreviations}

DLBCL, diffuse large-B-cell lymphoma; GCB DLBCL, germinal-center B-cell-like diffuse large-B-cell lymphoma; $A B C D L B C L$, activated $B$-cell-like diffuse large-B-cell lymphoma.

\section{Competing interests}

The authors declare that they have no competing interests.

\section{Authors' contributions}

All authors read and approved the final manuscript.

\section{Acknowledgements}

The authors thank Dr. Steven Suter, professor of clinical sciences and Dr. Matthew Breen from the College of Veterinary Medicine, North Carolina State University for the access to the dog lymphoma expression data.

\section{Author details}

${ }^{1}$ Dr. Su's Statistics, Department of Human Nutrition, Food, and Animal Sciences, University of Hawaii at Manoa, Honolulu, HI 96822, USA.

${ }^{2}$ Bioinformatics Research Center, North Carolina State University, Raleigh, NC 27695, USA. ${ }^{3}$ Biomarker and Predictive Analytics, GlaxoSmithKline, 5 Moore Drive, Research Triangle Park, NC 27709, USA. ${ }^{4}$ Department of Genetic, University of North Carolina, Chapel Hill, NC 27599, USA. ${ }^{5}$ Department of Clinical Sciences, Center for Comparative Medicine \& Translational Research, North Carolina State University, Raleigh, NC 27695, USA. ${ }^{6}$ Department of Molecular Biomedical Sciences, College of Veterinary Medicine, North Carolina State University, Raleigh, NC 27695, USA. ${ }^{7}$ Department of Statistics, North Carolina State University, Raleigh, NC 27695, USA.

Received: 9 December 2011 Accepted: 14 November 2012 Published: 5 January 2013

\section{References}

1. Lossos I, Czerwinski DK, Alizadeh AA, Wechser MA, Tibshirani R, Botstein D, Levy R: Prediction of survival in diffuse large-B-cell lymphoma based on the expression of six genes. N Engl J Med 2004, 350:1828-1837.

2. Lenz G, Staudt L: Aggressive lymphomas. N Engl J Med 2010, 362:1417-1429.

3. The International Non-Hodgkin's Lymphoma Prognostic Factors Project: A predictive model for aggressive non-Hodgkin's lymphoma. N Engl J Med 1993, 329:987-992.

4. Shipp MA, Ross KN, Tamayo P, Weng AP, Kutok JL, Aguiar RC, Gaasenbeek M, Angelo M, Reich M, Pinkus GS, Ray TS, Koval MA, Last KW, Norton A, Lister TA, Mesirov J, Neuberg DS, Lander ES, Aster JC, Golub TR: Diffuse large B-cell lymphoma outcome prediction by gene-expression profiling and supervised machine learning. Nat Med 2002, 8:68-74

5. Rosenwald A, Wright G, Chan WC, Connors JM, Campo E, Fisher RI, Gascoyne RD, Muller-Hermelink HK, Smeland EB, Giltnane JM, Hurt EM Zhao H, Averett L, Yang L, Wilson WH, Jaffe ES, Simon R, Klausner RD, Powell J, Duffey PL, Longo DL, Greiner TC, Weisenburger DD, Sanger WG, Dave BJ, Lynch JC, Vose J, Armitage JO, Montserrat E, Lpez-Guillermo A, et al.: The use of molecular profiling to predict survival after chemotherapy for diffuse large-B-cell lymphoma. N Engl J Med, 346:1937-1947.

6. Lenz G, Wright GW, Emre NC, Kohlhammer H, Dave SS, Davis RE, Carty S, Lam LT, Shaffer AL, Xiao W, Powell J, Rosenwald A, Ott G, Muller-Hermelink HK, Gascoyne RD, Connors JM, Campo E, Jaffe ES, Delabie J, Smeland EB, Rimsza LM, Fisher RI, Weisenburger DD, Chan WC, Staudt LM: Molecular 
subtypes of diffuse large B-cell lymphoma arise by distinct genetic pathways. Proc Nat Acad Sci USA 2008, 105:13520-13525.

7. Lenz G, Wright G, Dave SS, Xiao W, Powell J, Zhao H, Xu W, Tan B, Goldschmidt N, labal J, Vose J, Bast M, Fu K, Weisenburger DD, Greiner TC, Armitage JO, Kyle A, May L, Gascoyne RD, Connors JM, Troen G, Holte H, Kvaloy S, Dierickx D, Verhoef G, Delabie J, Smeland EB, Jares P, Martinez A, Lopez-Guillermo A, et al.: Stromal gene signatures in large-B-cell lymphomas. N Engl J Med 2008, 359:2314-2323.

8. Wright G, Tan B, Rosenwald A, Hurt EH, Wiestner A, Staudt LM: A gene expression-based method to diagnose clinically distinct subgroups of diffuse large B-cell lymphoma. Proc Nat Acad Sci USA 2003, 100:9991-9996.

9. Alizadeh AA, Eisen MB, Davis RE, Ma C, Lossos IS, Rosenwald A, Boldrick JC, Sabet H, Tran T, Yu X, Powell Jl, Yang L, Marti GE, Moore T, Hudson JJr, Lu L, Lewis DB, Tibshirani R, Sherlock G, Chan WC, Greiner TC, Weisenburger DD, Armitage JO, Warnke R, Levy R, Wilson W, Grever MR, Byrd JC, Botstein $D$, Brown PO, Staudt LM, et al.: Distinct types of diffuse large B-cell lymphoma identified by gene expression profiling. Nature 2000, 403:503-511.

10. Eisen M, Spellman PT, Brown PO, Botstein D: Cluster analysis and display of genome-wide expression patterns. Proc Nat Acad Sci USA 1998, 95:14863-14868.

11. Golub T, Slonim DK, Tamayo P, Huard C, Gaasenbeek M, Mesirov JP, Coller H, Loh ML, Downing JR, Caligiuri MA, Bloomfield CD, Lander ES: Molecular classification of cancer: class discovery and class prediction by gene expression monitoring. Science 1999, 286:531-537.

12. Blenk S, Engelmann J, Weniger M, Schultz J, Dittrich M, Rosenwald A, Mller-Hermelink HK, Mller T, Dandekar T: Germinal center B cell-like $(G C B)$ and activated $B$ cell-like $(A B C)$ type diffuse large $B$ cell lymphoma (DLBCL): analysis of molecular predictors, signatures, cell cycle state and patient survival. Cancer Inform 2007, 3:399-420.

13. Smyth G: Linear models and empirical Bayes methods for assessing differential expression in microarray experiments. Stat App/ Genet Mol Biol 2004, 3:3.

14. Sonnhammer E, Koonin E: Orthology, paralogy and proposed classification for paralog subtypes. TIG 2002, 18:619-620.

15. McLachlan G, Basford K: Mixture models: inference and applications to clustering. New York: Marcel Dekker; 1988.

16. McLachlan G, Peel D: Finite mixture models. New York: Wiley; 2000.

17. Efron B: Bootstrap methods: another look at the jackknife. Ann Stat 1979, 7:1-26

18. Efron B: Better bootstrap confidence intervals. J Am Stat Assoc 1987, 82:171-185.

19. Barrett T, Troup DB, Wilhite SE, Ledoux P, Rudnev D, Evangelista C, Kim IF, Soboleva A, Tomashevsky M, Marshall KA, Phillippy KH, Sherman PM, Muertter RN, Edgar R: NCBI GEO: archive for high-throughput functional genomic data. Nucl Acids Res 2009, 37:D885-D890.

20. Lachenbruch P, Mickey M: Estimation of error rates in discriminant analysis. Technometrics 1968, 10:1-11.

21. Benjamini $Y$, Hochberg $Y$ : Controlling the false discovery rate: a practical and powerful approach to multiple testing. $J$ Roy Statist SOC Ser B 1995, 57:289-300.

22. MacQueen J: Some methods for classification and analysis of multivariate observations. In Proceedings of Fifth Berkeley Symposium on Math Statistics and Probability. Statistics. Berkeley: 1965 June 21-July 18, Statistical Laboratory of the University of California; 19651:281-297.

23. SAS: SAS onlineDoc ${ }^{\oplus}$ 9.1.3. (2002-2008) Available at http://support.sas. com/onlinedoc/913.

24. Dempster A, Laird N, Rubin D: Maximum likelihood from incomplete data via the EM algorithm. J Roy Statist Soc Ser B 1977, 39:1-38.

25. Kaplan E, Meier $P$ : Nonparametric estimation from incomplete observations. J Am Stat Assoc 1958, 53:457-481.

26. Mantel N: Evaluation of survival data and two new rank order statistics arising in its consideration. Cancer Chemother Rep 1966, 50:163-170.

27. Maglott D, Ostell J, Pruitt KD, Tatusova T: Entrez Gene: gene-centered information at NCBI. Nucl Acids Res 2007, 35:D26-D31.

28. Spanevello R, Mazzanti CM, Schmatz R, Thom G, Bagatini M, Correa M, Rosa C, Stefanello N, Bell LP, Moretto MB, Oliveira L, Morsch VM, Schetinger MR: The activity and expression of NTPDase is altered in lymphocytes of multiple sclerosis patients. Clin Chim Acta 2009, 411:210-214.
29. Behrens T, Kearns GM, Rivard JJ, Bernstein HD, Yewdell JW, Staudt LM: Carboxyl-terminal targeting and novel post-translational processing of JAW1, a lymphoid protein of the endoplasmic reticulum. J Biol Chem 1996, 271:23528-23534.

30. Tedoldi S, Paterson JC, Cordell J, Tan SY, Jones M, Manek S, Dei Tos, A P, Roberton H, Masir N, Natkunam Y, Pileri SA, Facchetti F, Hansmann ML, Mason DY, Marafioti T: Jaw1/LRMP, a germinal centre-associated marker for the immunohistological study of B-cell lymphomas. J Pathol 2006, 209:454-463.

31. Natkunam Y, Zhao S, Mason DY, Chen J, Taidi B, Jones M, Hammer AS, Hamilton Dutoit, S, Lossos IS, Levy R: The oncoprotein LMO2 is expressed in normal germinal-center $B$ cells and in human B-cell lymphomas. Blood 2007, 109:1636-1642.

32. Morton LM, Purdue MP, Zheng T, Wang SS, Armstrong B, Zhang $Y$, Menashe I, Chatterjee N, Davis S, Lan Q, Vajdic CM, Severson RK, Holford TR, Kricker A, Cerhan JR, Leaderer B, Grulich A, Yeager M, Cozen W, Hoar Zahm, S, Chanock SJ, Rothman N, Hartge P: Risk of non-Hodgkin lymphoma associated with germline variation in genes that regulate the cell cycle, apoptosis, and lymphocyte development. Cancer Epidemiol Biomarkers Prev 2009, 18:1259-1270.

33. Durnick D, Law ME, Maurer MJ, Natkunam Y, Levy R, Lossos IS, Kurtin PJ, MCPhail ED: Expression of LMO2 is associated with $t(14 ; 18) / / G H-B C L 2$ fusion but not BCL6 translocations in diffuse large B-cell lymphoma. Am J Clin Path 2010, 134:278-281.

34. Han J, Huang NN, Kim DU, Kehrl JH: RGS1 and RGS13 mRNA silencing in a human $B$ lymphoma line enhances responsiveness to chemoattractants and impairs desensitization. J Leukoc Bio/ 2006, 79:1357-1367.

35. Banham A, Connors JM, Brown PJ, Cordell JL, Ott G, Sreenivasan G, Farinha P. Horsman DE, Gascoyne RD: Expression of the FOXP1 transcription factor is strongly associated with inferior survival in patients with diffuse large B-cell lymphoma. Clin Cancer Res 2005, 11:1065-1072.

36. Goatly A, Bacon CM, Nakamura S, Ye H, Kim I, Brown PJ, Ruskon-Fourmestraux A, Cervera P, Streubel B, Banham AH, Du MQ: FOXP1 abnormalities in lymphoma: translocation breakpoint mapping reveals insights into deregulated transcriptional control. Mod Pathol 2008, 21:902-911.

37. Korac $P$, Dominis, $M:$ Prognostic markers and gene abnormalities in subgroups of diffuse large B-cell lymphoma: single center experience. Clin Sci 2008, 49:618-624.

38. Nyman H, Jerkeman M, Karjalainen-Lindsberg ML, Banham AH, Leppä S: Prognostic impact of activated B-cell focused classification in diffuse large B-cell lymphoma patients treated with R-CHOP. Mod Pathol 2009, 22:1094-1101.

39. Tzankov A, Zlobec I, Went P, Robl H, Hoeller S, Dirnhofer S: Prognostic immunophenotypic biomarker studies in diffuse large $B$ cell lymphoma with special emphasis on rational determination of cut-off scores. Leuk Lymphoma 2010, 21:902-911.

doi:10.1186/1479-7364-7-2

Cite this article as: Su et al.: Gene selection and cancer type classification of diffuse large-B-cell lymphoma using a bivariate mixture model for twospecies data. Human Genomics 2013 7:2. 